DOI: $10.21892 / 978-958-5547-65-0.8$

\title{
8. Diseño de productos sustentables para la agricultura familiar
}

Diego Alejandro Piracoca-Chaves ${ }^{1}$

Rosalba Frías-Navarro ${ }^{2}$

\section{Introducción}

El propósito de este escrito es despertar en los lectores la necesidad de pensar, desde sus propias disciplinas, intereses y quehaceres, en cómo contribuir con que las personas vulnerables de la sociedad puedan alcanzar su "vida querida". El enfoque, en este caso particular, es hacia la propuesta de soluciones para que los campesinos puedan mejorar sus condiciones de vida a través de la creación de diseños sustentables innovadores, que contribuyan con ingresos económicos adicionales para los productos agrícolas producidos en granjas de agricultura familiar sustentable.

En cuanto a los proyectos productivos, el concepto de la vida querida adquiere un significado especial, y como lo menciona De Roux Rengifo S.J., (2010, p. 233), lo que se trata es de construir de forma colectiva "la manera de vivir que la gente quiere vivir. Es decir, construir las condiciones para proteger y expresar la grandeza de la dignidad humana como lo quiere un pueblo con sus tradiciones, su sensibilidad, su medio ambiente y sus sueños".

Para la FAO (2017), más del 90\% de los 570 millones de granjas agrícolas del mundo cuentan con una gestión individual o familiar y producen el $80 \%$ de los alimentos en términos de valor. Estas familias se dedican a la agricultura, pesca, trabajo en bosques, o cría de aves o ganado, por tanto, tienen un gran potencial para la lucha contra la pobreza y el hambre y el desarrollo sostenible de los territorios rurales. Los agricultores familiares son actores fundamentales para el desarrollo rural, ya que como

1 Diseñador Industrial de la Pontificia Universidad Javeriana.

2 Ingeniera Agrícola de la Universidad Nacional de Colombia. Magíster en Administración de la Universidad Nacional de Colombia. Doctora en Ingeniería, Industria y Organizaciones de la Universidad Nacional de Colombia. 
están vinculados a las redes y a la cultura del territorio por medio de su participación e intercambio en los mercados locales, contribuyen con la generación de empleos agrícolas y no agrícolas en las regiones

Cabe mencionar, además de la potencialidad de la agricultura familiar, los retos y dificultades que enfrentan este tipo de productores: pobreza, exclusión y condiciones desfavorables para el acceso a salud y educación; los limitantes para el desarrollo mismo de los sistemas productivos, como el bajo capital de trabajo debido a los escasos recursos económicos, la tenencia de la tierra o la informalidad en la propiedad rural, la falta de vías para transportar los productos, carencia de asistencia técnica, el cambio climático, los altos costos de producción; y a lo anterior, en algunas zonas del país se produce un cambio de la economía campesina tradicional, para dar paso a cultivos ilícitos destinados a mercados internacionales. Lo expuesto, sumado a las limitadas oportunidades en el campo, incide en la alta tasa de migración hacia las ciudades y en la falta de mano de obra en el sector rural.

Es en este contexto de potencialidades y limitantes para la agricultura familiar, en donde se puede aportar desde una propuesta de diseño un elemento diferencial, que permita que los campesinos puedan mejorar sus condiciones de vida, incrementando sus ingresos a través de modelos innovadores de productos que contribuyan con la economía familiar. Para tal fin, el presente trabajo de investigación se inicia a partir del estudio de desarrollar una propuesta de diseño de empaques biodegradables utilizando como materia prima los desechos del cultivo de café. A partir de la realización de un estado del arte sobre el tema empaques biodegradables con fuentes orgánicas diferentes al PLA(Acido Poli Láctico), se encontró información que presenta como materiales posibles a usar a las cáscaras de plátano, desechos del café y a la fermentación de bacterias para producir PHA (Polihroxialcanoato) en la construcción de materiales biodegradables (Piracoca \& Frias, 2016, pp. 597-505).

En este documento se presenta una propuesta de diseño de producto sustentable exponiendo un ejemplo desarrollado en un ambiente académico: el proceso de investigación de un estudiante para lograr el diseño de un empaque amigable con el medio ambiente. Este se comenzó a desarrollar en un Semillero de Investigación, en el cual un estudiante de Diseño Industrial fue motivado por su docente hacia el desarrollo de una investigación en su 
disciplina en el marco de la sustentabilidad. La pregunta de investigación que se planteó dicho estudiante fue cómo desarrollar empaques biodegradables $y$, a medida que ha ido profundizando en su investigación se ha centrado en el diseño de empaques a partir de residuos de cultivos y en la forma como los pequeños productores pueden producirlos directamente en los lugares de origen.

\section{Diseño de un empaque sustentable a partir del aprovechamiento de los desechos del cultivo de café}

Según Geonova (2011), "un producto sustentable es aquel que durante toda su cadena de producción utiliza los recursos naturales, humanos y económicos de la manera más eficiente, inteligente y responsable, asegurando que las generaciones futuras puedan disfrutar de, al menos, lo mismo que nosotros tenemos ahora". Para Jedlicka (2009, p. 191) (citado por Bravo (2015, p. 97)), un producto es sustentable cuando es beneficioso, seguro y saludable para las personas y las comunidades a lo largo de su ciclo de vida, cumple con los criterios de rendimiento y costos del mercado y utiliza energía renovable favoreciendo la reutilización de recursos. Bravo (2015) aplica la anterior definición en el diseño de empaques, los que por taxonomía se consideran como un producto.

El desarrollo de productos desde el diseño industrial está ligado al análisis de las necesidades de una población, las cuales son transformadas en "inputs o entradas" para dar comienzo al proceso de diseño. De modo que, con una serie de comprobaciones con el usuario e interacciones con las propuestas realizadas, se afina o robustece el producto, con el objetivo de ser lanzado al mercado. En el caso del diseño de un producto sustentable, además se deben considerar las dimensiones: ambiental, social y económica en el proceso (Aguayo, 2013). En cuanto a la dimensión ambiental, uno de los grandes problemas es respecto a la degradabilidad de los materiales, caso concreto de los polímeros que por sus propiedades pueden superar los 300 años (Medina, 2005).

Paralelamente mientras se produce este impacto negativo al medio ambiente, también a nivel macro crece la acumulación de estos "desechos" hasta el punto de generar una isla en el Pacífico Norte, su extensión actualmente alcanza los $148.827 \mathrm{Km}$ cuadrados, sin dimensionar su expansión diaria. La huella de dicha situación puede agravarse a tal punto de 
alcanzar la dimensión igual o superior a la de un continente (MDZ, 2017). Todo si se sigue con el consumismo que el mercado promueve, sin contar, la restante incidencia durante los procesos de extracción y fabricación, los cuales generan grandes concentraciones de dióxido de carbono dirigido hacia la atmósfera y aumentan el calentamiento global.

Las dimensiones: social y económica se abarcan en este caso de estudio no solo desde su relevancia concebida desde la sostenibilidad caso de la estrategia de innovación fractal: yacimiento del valor, la ingeniería sostenible (Aguayo, 2013) hasta la triple bottom line (McDonough, 2002) sino también, trascender al contexto de lo rural con el enfoque de la recuperación de la economía local. De acuerdo con Latouche \& Harpagès (2011, p. 78), "la autonomía económica local implica orientarse hacia la búsqueda de una autosuficiencia alimentaria y energética pero también hacia una autonomía de financiación que permita el cumplimiento de proyectos locales artesanales, industriales y de servicios". En línea con lo anterior, el proceso de diseño de empaque por proponer, debe cumplir no solamente con las necesidades de una población, sino que además, este producto debe poderse producir directamente en el lugar donde se dispone de la materia prima: los desechos de café.

De acuerdo con lo anteriormente expuesto, se busca que la propuesta de producto sustentable pueda alivianar las problemáticas ambientales, sociales y económicas, de este modo, los campesinos productores tengan la posibilidad de desarrollar la(s) propuesta(s) en sus territorios sin depender de un gran capital para su fabricación. Estos factores de sostenibilidad económica y social permitirán generar una fuente capital adicional con el fin de promover equidad y mejores condiciones de vida.

\section{El proceso de diseño}

En la Tabla 1 se presenta la información relativa al concepto de diseño, requerimientos y determinantes de producto desde una visión de diseño industrial. Estos factores tienen como función encaminar la propuesta hacia los aspectos necesarios de producción, función, usabilidad y material del producto, y de esta forma, la planificación desde un principio es clara, lo que es fundamental para cuando se empiecen a realizar los respectivos diseños. 
Tabla 1

Concepto de diseño, requerimientos y determinantes

Concepto de proyecto: Desarrollar una línea de productos a partir del aprovechamiento de los desechos orgánicos de la cadena productiva del café y desechos de papelería para su respectiva fabricación en lugares propios de la agricultura, generando un producto sostenible con el medio ambiente.

Concepto de producto: Generar un producto enfocado al embalaje para proporcionar protección y seguridad de los productos transportados.

Concepto de producto: Realizar un producto enfocado al consumo del café, para que este mejore el momento de la actividad, reduciendo una huella negativa sobre el ambiente.

Concepto de diseño: Diseñar un separador para los vasos de vidrios que será usado para el proceso de embalaje, de modo que, este separador ayudará a distribuir el producto más fácilmente en cajas y protegerá el producto en su totalidad de posibles daños.

Concepto de diseño: Desarrollar un protector para vasos de café que aislé el calor generado por la bebida y de este modo, la actividad de consumo se vea beneficiada en términos de usabilidad.

\begin{tabular}{|l|l|}
\hline \multicolumn{1}{|c|}{ Requerimientos (1) } & \multicolumn{1}{|c|}{ Determinantes (1) } \\
\hline & $\begin{array}{l}\text { El separador protege a los } \\
\text { vasos de vidrio de posibles } \\
\text { golpes que puedan romper el } \\
\text { envase. } \\
\text { El material del producto es desarrollado a a } \\
\text { partir de materias primas recicladas. }\end{array}$ \\
$\begin{array}{l}\text { El separador proporciona } \\
\text { condiciones óptimas de } \\
\text { embalaje del producto. } \\
\text { es de } 0,1 \mathrm{~cm} .\end{array}$ & $\begin{array}{l}\text { La fabricación del producto } \\
\text { debe ser desarrollada en fincas } \\
\text { caficulturas. } \\
\text { La fabricación del material del } \\
\text { producto debe ser desarrollada } \\
\text { en fincas caficultoras. }\end{array}$ \\
\hline
\end{tabular}




\begin{tabular}{|c|c|}
\hline Requerimientos (1) & Determinantes $(1)$ \\
\hline $\begin{array}{l}\text { El producto separa de forma uniforme a los } \\
\text { productos ahorrando la mayor cantidad de } \\
\text { espacio posible }\end{array}$ & $\begin{array}{l}\text { El producto permite un } \\
\text { apilamiento sencillo que no } \\
\text { ocupa gran espacio. } \\
\text { El producto debe ser } \\
\text { desarrollado con la tecnología } \\
\text { existente en fincas caficultoras. }\end{array}$ \\
\hline Requerimientos (2) & Determinantes $(2)$ \\
\hline $\begin{array}{l}\text { El material del producto es desarrollado a } \\
\text { partir de materias primas recicladas. } \\
\text { El espesor que posee cada lámina del separador } \\
\text { es de } 0,1 \mathrm{~cm} \text {. } \\
\text { El producto aísla una temperatura de } 80^{\circ} \mathrm{c} \\
\text { El producto protege la palma de la mano y a } \\
\text { los dedos. }\end{array}$ & $\begin{array}{l}\text { La fabricación del material del } \\
\text { producto debe ser desarrollada } \\
\text { en fincas caficultoras. } \\
\text { El producto permite un } \\
\text { apilamiento sencillo que no } \\
\text { ocupa gran espacio. } \\
\text { El producto debe ser } \\
\text { desarrollado con las } \\
\text { tecnologías existentes en } \\
\text { fincas caficultoras. }\end{array}$ \\
\hline
\end{tabular}

Fuente: elaboración propia.

\section{Propuesta: separador}

La siguiente propuesta de diseño tiene como objetivo aprovechar residuos orgánicos para el desarrollo de productos para la industrial, en este caso, separadores de envases, los cuales, son usados actualmente para los procesos de embalaje. Según el mercado y en comparación con la propuesta no hay ningún cambio formal en el diseño, más sin embargo su diferenciador radica en la materia prima para desarrollar el material. Esto, debido a que en los actuales se está utilizando el cartón micro corrugado. Este separador estaría hecho de papel reciclado y a partir de insights-inputs orgánicos.

Avanzando con el tema del diseño, la propuesta de la Figura 1 se realizó a partir de un envase presente en el mercado colombiano, de allí se sacaron algunas de sus medidas, estas fueron $7 \mathrm{~cm} \times 7 \mathrm{~cm} \times 13 \mathrm{~cm}$. Conviene señalar que el diseño realizado no es exclusivo y puede variar también dependiendo de las medidas de los distintos envases de café existentes en el mercado. La estructura es dada por el ensamble de media caja, este 
funciona a través del encaje de dos pestañas, las cuales poseen ranuras a lados contrarios para mejorar la resistencia de compresión de las láminas ensambladas (Bernal \& Ticora, 2008, p. 41).

Como bien se había mencionado dentro de los requerimientos de diseño, este producto tendrá un espesor de un milímetro, como también unas dimensiones para la primera pestaña de $72,7 \mathrm{~cm} \times 13,5 \mathrm{~cm}$ y para la segunda de $63,6 \mathrm{~cm} \times 13,5 \mathrm{~cm}$. En la Figura 2, se encuentran las medidas técnicas del producto en relación a los envases de vidrio de café, los cuales tienen una tolerancia de $1,1 \mathrm{~mm}$, porque entre más juntos estén se evitará en mayor posibilidad un choque de envases y a causa de esto se pueda llegar a romper o llegar en las condiciones menos ideales para ser exhibido en una góndola.
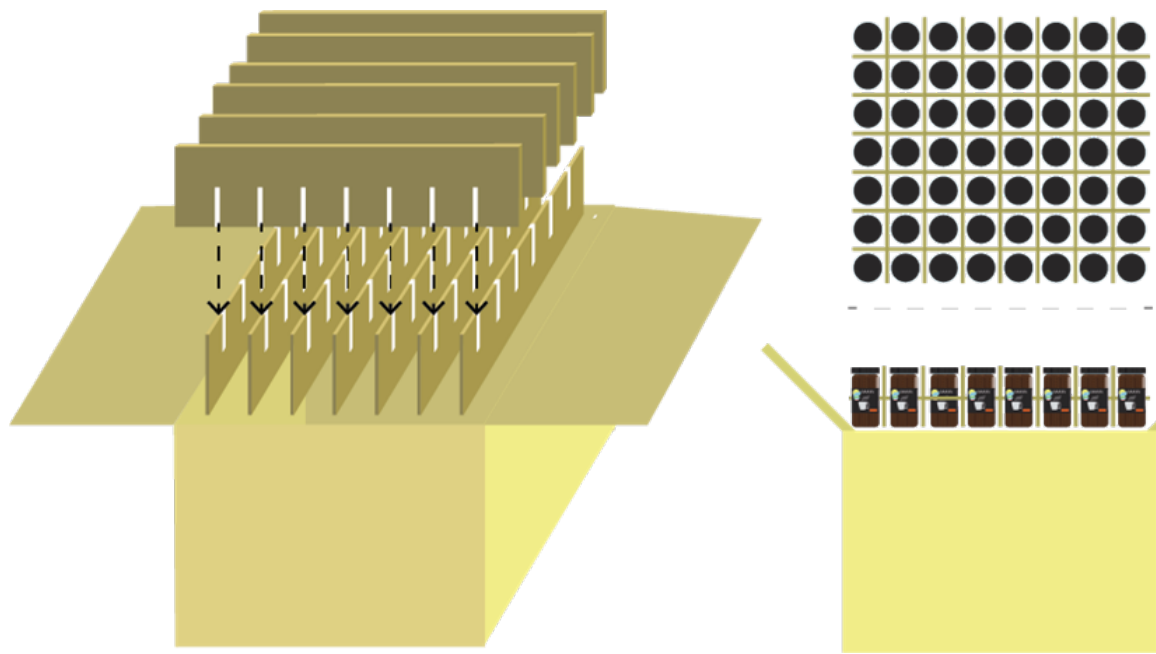

Figura 1. Diseño y configuración del separador con los envases.

Fuente: elaboración propia. 


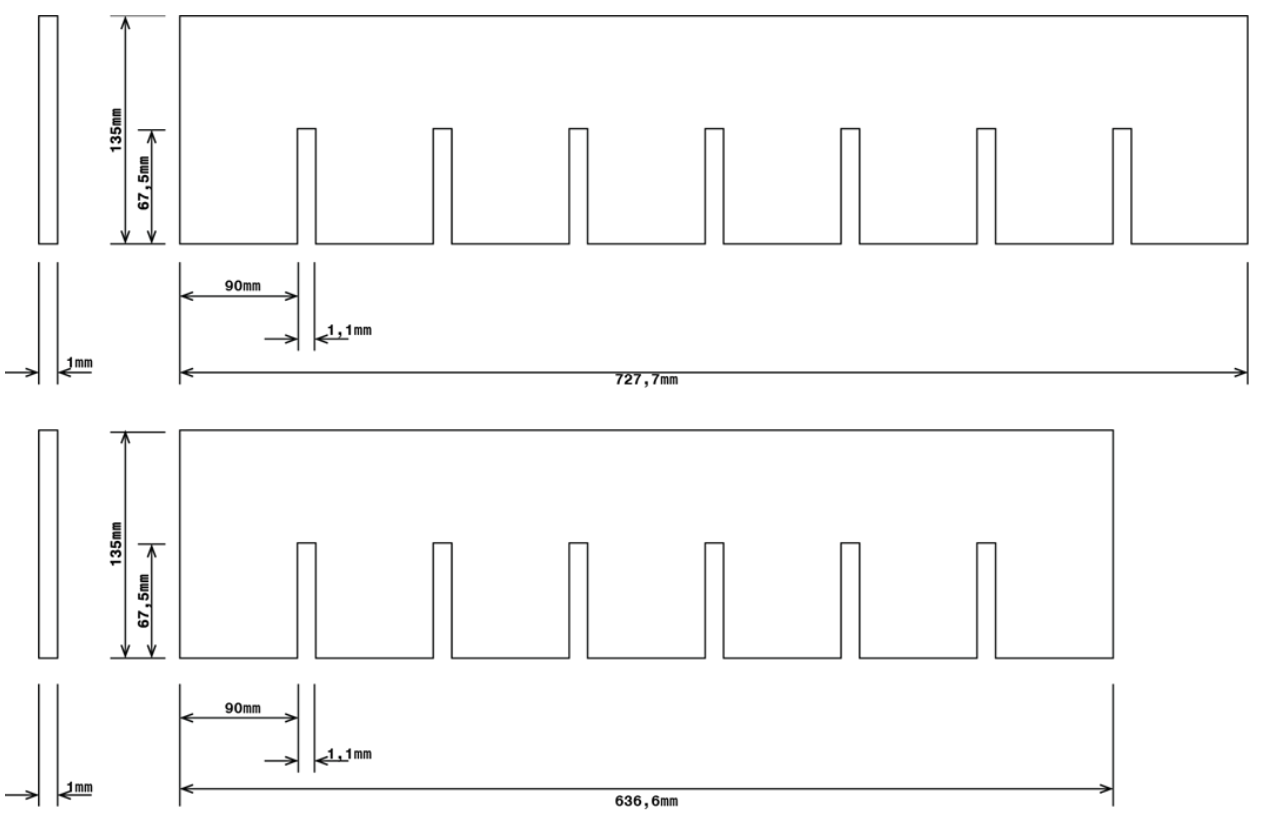

Figura 2. Plano del separador

Fuente: elaboración propia.

\section{Propuesta: protector de vaso}

La siguiente propuesta, como se indicó en los conceptos y los requerimientos de diseño, es un protector de vaso, el cual se plantea desde la variabilidad de aplicaciones para el papel reciclable. De esta manera, una de esas propuestas es un subproducto usado en grandes cantidades al vender un moca, un café con leche o un cappuccino, etc., y evita pequeñas quemaduras en el consumidor que lo beba, se queme. En el mercado actual se pueden encontrar en las grandes empresas comercializadoras de café el uso de este producto como en Juan Valdez, Starbucks, Oma, Scooters Coffee, entre otras más.

Por otro lado, este protector solo se encuentra en cartón y en una única versión formal dentro del mercado de la comercialización de bebidas calientes, específicamente café. Por otra parte, existe una variación de cartón que están empezando a implementar algunas compañías, caso de Corrucol, en donde el material es 100\% reciclado, con lo cual, deja a esta propuesta en iguales situaciones de competencia. Entonces, por este hallazgo, se analiza el actual protector utilizado por las compañías comercializadoras 
de café. Lo anterior arroja ciertos descubrimientos, estos permiten mejorar la función de este producto en términos de usabilidad.

La prueba aplicada para analizar este producto fue empírica, bajo un protocolo de pensamiento manifestado (Rincón, 2017, p. 166), este consiste en que el usuario de prueba consuma cualquier bebida de café y mientras ejecuta cada paso de la secuencia de uso, va manifestando algunas inconformidades o satisfacciones con el producto. Para resumir esta prueba, en términos de percepción por los "productos verdes" los usuarios manifiestan: el hecho de ser 100\% reciclado lo hace más atractivo y también, posiciona a la empresa como responsable con el medio ambiente. En otras palabras, son estrategias para la fidelización del consumidor como diferenciadores o potencializadores para acrecentar la demanda del producto.

En términos de dimensiones, manifiesta que este es pequeño, porque el dedo índice o meñique o los dos quedan por fuera de contacto con este protector (en la Figura 3, se da una representación de las inconformidades señaladas por el usuario). Adicionalmente, en temas de uso durante la actividad se determinan a los primeros 10 minutos como aquellos en los cuales la bebida comprada se encuentra y percibe a una temperatura muy alta. Por lo tanto, por medio del proceso de conducción de temperatura, el calor es transmitido a los dedos índice o meñique o los dos, causando una pequeña quemadura sobre la piel, esto lo señala como el aspecto más deficiente del producto y, respecto a los demás momentos de consumo no se halla otra insatisfacción o satisfacción relevante para la actividad. 


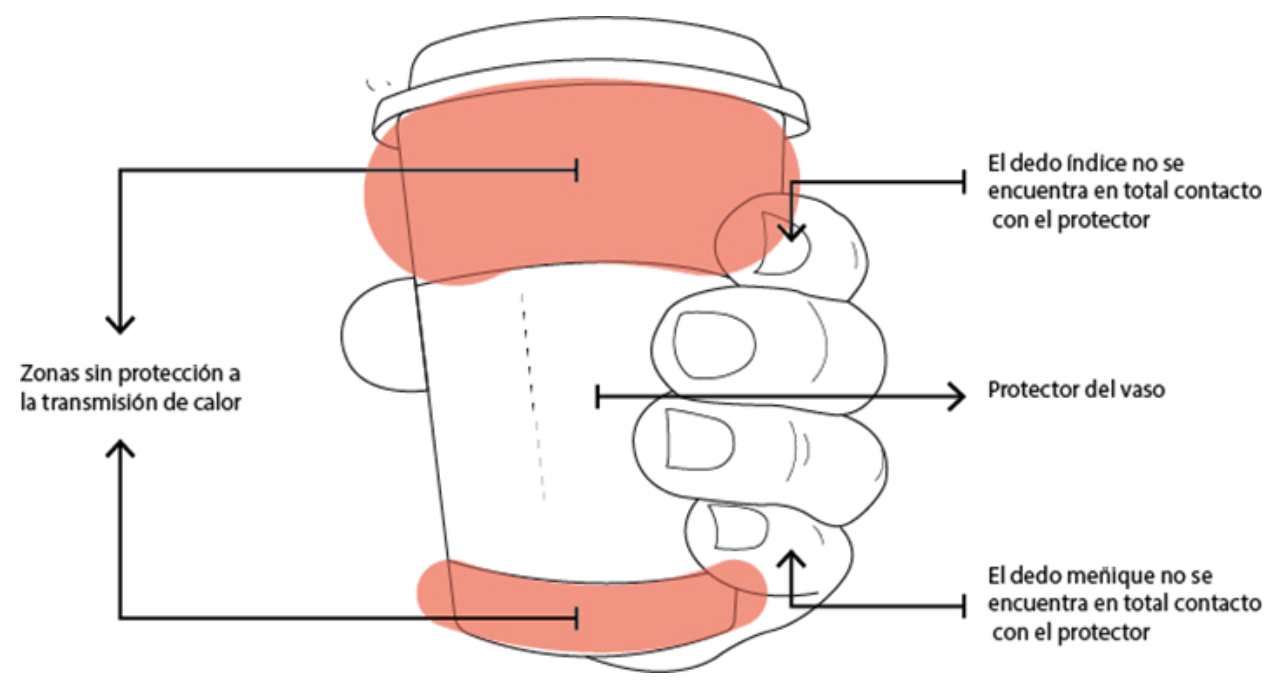

Figura 3. Representación de las problemáticas del producto que se encuentra actualmente en el mercado

Fuente: elaboración propia.

Aludiendo al anterior párrafo, bajo la dirección de los autores, en la Tabla 2 se muestra una caracterización de la propuesta planteada para este artículo y de la evaluada (actual), para que de este modo se puedan ver las ventajas y desventajas de cada uno. 
Tabla 2

Ventajas y desventajas de la propuesta como del protector evaluado.

\begin{tabular}{|c|c|c|c|}
\hline \multicolumn{2}{|c|}{ Protector propuesto } & \multicolumn{2}{|c|}{ Protector evaluado } \\
\hline Ventajas & Desventajas & Ventajas & Desventajas \\
\hline \multirow{5}{*}{$\begin{array}{l}\text { Material: } \\
100 \% \text { reci- } \\
\text { clado con } \\
\text { un 10\% de } \\
\text { sustancia } \\
\text { orgánica. }\end{array}$} & \multirow{10}{*}{$\begin{array}{c}\text { Proceso de } \\
\text { fabricación: } \\
\text { Artesanal } \\
\text { Tiempo } \\
\text { estimado de } \\
\text { producción: } \\
\text { Lento }\end{array}$} & Proceso de fabricación: & \multirow{8}{*}{$\begin{array}{l}\text { Usabilidad: No hay } \\
\text { protección en el } \\
\text { dedo índice como } \\
\text { meñique. } \\
\text { Dimensiones: No se } \\
\text { ajusta a las medidas } \\
\text { de la mano. }\end{array}$} \\
\hline & & Industrial. & \\
\hline & & Material: $100 \%$ & \\
\hline & & reciclado. & \\
\hline & & Percepción & \\
\hline \multirow{3}{*}{$\begin{array}{l}\text { Usabilidad: } \\
\text { Protección } \\
\text { total en los } \\
\text { dedos. }\end{array}$} & & $\begin{array}{l}\text { del producto: } \\
\text { ambientalmente }\end{array}$ & \\
\hline & & responsable. & \\
\hline & & Capacidad de & \\
\hline \multirow{2}{*}{$\begin{array}{l}\text { Percepción del } \\
\text { producto: Am- } \\
\text { bientalmente } \\
\text { responsable. }\end{array}$} & & $\begin{array}{l}\text { aislamiento termico. } \\
\text { Alto. }\end{array}$ & \\
\hline & & $\begin{array}{l}\text { Tiempo estimado de } \\
\text { producción: Rápido }\end{array}$ & \\
\hline \multicolumn{4}{|l|}{$\begin{array}{l}\text { Dimensión: } \\
\text { Dimensiones } \\
\text { ajustadas a los } \\
\text { dedos. }\end{array}$} \\
\hline $\begin{array}{l}\text { Capacidad de } \\
\text { aislamiento } \\
\text { térmico: alto. }\end{array}$ & & & \\
\hline
\end{tabular}

Fuente: elaboración propia.

Con base en cada una de las desventajas encontradas en el producto evaluado (Figura 4), se dispone de un proceso de bocetación, el cual arroja unas propuestas (Figura 5) que responden al concepto, los requerimientos y determinantes de diseño. Cada una de estas propuestas soluciona los problemas encontrados en la prueba empírica, como lo son el ajuste a la mano del usuario y la protección térmica a todos los dedos. Es decir, con el rediseño del producto se busca dar un nuevo valor agregado a este. De este modo, desde el planteamiento de este tipo de productos sostenible se está dando mejora a aspectos formales como de usabilidad y asimismo, generando un impacto positivo desde los ambiental, económico y social. 
Diseño de productos sustentables para la agricultura familiar

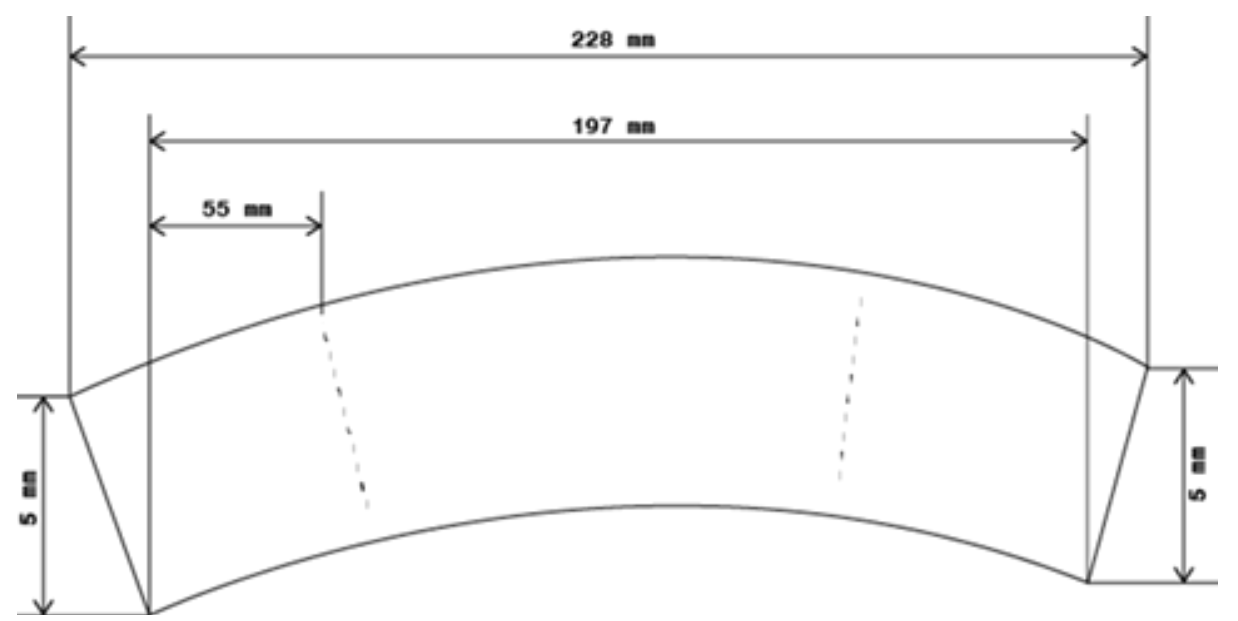

Figura 4. Dimensiones del protector evaluado. Fuente: elaboración propia.
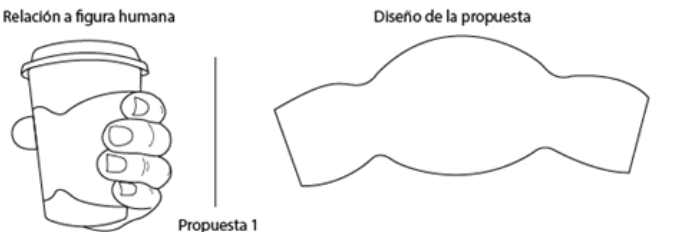

Comparacion fromal de los dos productos
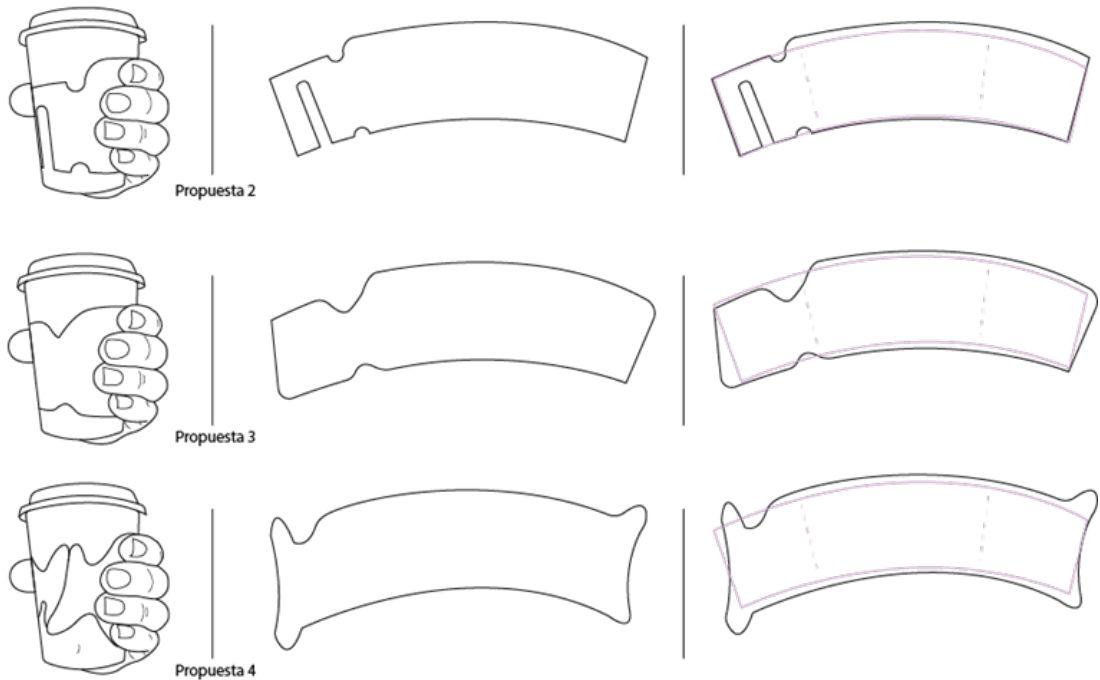

Figura 5. Propuestas del protector para vasos.

Fuente: elaboración propia. ${ }^{3}$

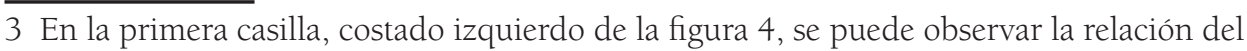




\section{Producción}

Con base a los requerimientos de diseño y el enfoque ambiental de las propuestas, el proceso productivo que se plantea para los dos diseños es el mismo, el cual es el licuado del papel reciclado y de algún otro elemento orgánico por disponer o agregar. En primer lugar, se toma como referencia la cadena productiva del café, (Figura 6) (García \& Olaya, 2006, pp. 197217) la finalidad de esta figura es observar e identificar cada uno de sus desechos dependiendo del proceso que lo antecede y procede y de este modo, clasificarlos en la Tabla 3, la cual contiene la descripción breve de cada uno junto a su proceso de procedencia.

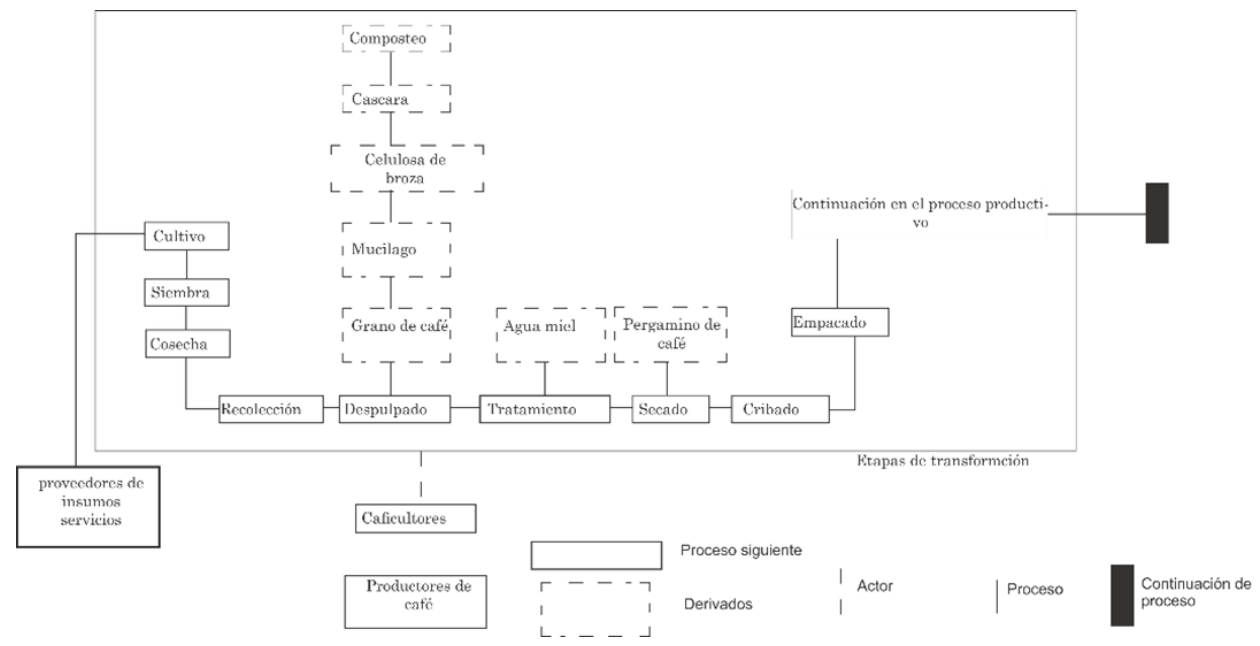

Figura 6. Cadena productiva del café

Fuente: Construcción de los autores (Piracoca \& Frias, 2016, p. 503) basado en (Gutiérrez, Henao, \& Oviedo, 2014, pp. 23-26) \& (García \& Olaya, 2006, p. 202) Grafico 1. Cadena productiva del café.

El método de clasificación y caracterización fue determinado de manera experimental, por razones relacionadas a la cantidad y calidad de la información hallada tanto en bases de datos como en internet sobre cada uno de estos desechos. Dicho contenido es desarrollado a partir de los

producto con la figura humana. En la casilla del medio de la figura 4, se puede visualizar el diseño del producto. El contorno de la forma que se alcanza a ver es la misma que se debe recortar para el proceso de fabricación y dé como resultado la simulación que se ve a mano izquierda. Y, en la tercera casilla, costado derecho de la figura 4, se puede observar la comparación formal como de dimensiones de los dos productos, demostrando los cambios que se plantearon para cada propuesta. 
siguientes apartados dentro de la casilla de "descripción": el estado de la materia del desecho, la finalidad o el pos uso que actualmente se aplica y la composición de este.

\section{Tabla 3}

Desechos de la cadena productiva del café.

\begin{tabular}{|c|c|c|}
\hline Proceso & Salidas & Descripción \\
\hline \multirow{10}{*}{ Despulpado } & & Estado: Sólido \\
\hline & Grano de café & $\begin{array}{l}\text { Finalidad: Es la salida más importante de todo } \\
\text { el proceso porque es el producto que se usará } \\
\text { para la preparación de la bebida. Uno de sus } \\
\text { componentes es la pulpa, la cual es utilizada } \\
\text { también para la lombricultura. }\end{array}$ \\
\hline & \multirow{3}{*}{ Mucílago } & Estado: Líquido \\
\hline & & $\begin{array}{l}\text { Composición: Agua, azucares y sustancias } \\
\text { pécticas }\end{array}$ \\
\hline & & $\begin{array}{l}\text { Finalidad: Mantener en condiciones óptimas al } \\
\text { fruto esto depende de las lluvias del ambiente, lo } \\
\text { cual hace que madure más rápido o no. }\end{array}$ \\
\hline & \multirow{3}{*}{ Celulosa de broza } & Estado: Líquido \\
\hline & & Composición: Azúcares \\
\hline & & $\begin{array}{l}\text { Finalidad: Abono orgánico, complemento de } \\
\text { alimento animal o mejorador de los suelos }\end{array}$ \\
\hline & \multirow{2}{*}{ Cáscara } & Estado: Sólido \\
\hline & & Finalidad: Abono \\
\hline \multirow[b]{3}{*}{ Tratamiento } & \multirow[b]{3}{*}{ Agua miel } & Estado: Líquido \\
\hline & & $\begin{array}{l}\text { Composición: Agua, azucares y sustancias } \\
\text { pécticas }\end{array}$ \\
\hline & & $\begin{array}{l}\text { Finalidad: Mantener en condiciones óptimas al } \\
\text { fruto, como también después de los dos lavados } \\
\text { respectivos que se realizan al fruto puede ser una } \\
\text { buena mezcla con el compost }\end{array}$ \\
\hline \multirow{2}{*}{ Secado } & \multirow{2}{*}{ Pergamino de café } & Estado: Sólido \\
\hline & & Finalidad: Fuente energética en calderas \\
\hline
\end{tabular}

Fuente: elaboración propia basada en (Anacafé.; Anacafé.; Puerta \& Ríos, 2011, pp. 23-40). 
A partir de la Tabla 3 se tiene una guía para realizar una elección del desecho orgánico que se usaría para el desarrollo del material. Para comenzar, el grano de café no puede ser usado para este producto, porque como se menciona en su finalidad, es el elemento principal para preparar la bebida y es el insumo económico principal de los campesinos productores. El mucílago es caracterizado como un elemento principalmente viscoso, además, su composición está contiene en su mayoría azucares y sustancias pécticas, es decir, si esta sustancia se mezcla con el papel reciclado, el material resultante tendrá cambios en sus propiedades físicas como mecánicas.

Por el caso de la celulosa de broza y del agua miel se cuentan con el mismo planteamiento de las propiedades del mucílago. Por otra parte, con las salidas sólidas, en primera observación, la cáscara al licuarla con el papel reciclado y homogenizarla puede contener algunos líquidos viscosos en las paredes como en el interior, esto hace más difícil el proceso de volver uniforme la mezcla y por consiguiente, al requerir más tiempo como esfuerzo por parte de las cuchillas se generará un mayor consumo de energía, asimismo se crea a un gasto económico para la familia como en la producción en términos del gasto mecánico a estas hojas.

Por último, tras observar en la información recolectada, el estado posterior del pergamino de café al proceso de secado, genera un material frágil, el cual puede llegar a mezclase de manera efectiva en el proceso de homogenización con el papel reciclado. Con lo anterior, se determina a esta opción como la más ajustable al requerimiento del material. No obstante, si este aprovechamiento se contrapone al actual, en donde se utiliza como fuente de calderas, esto crea emisiones nocivas al ambiente como a las personas. En otras palabras, el factor de sostenibilidad ayuda a determinar a esta utilización del desecho como más beneficiosa para el estado actual de las entradas y salidas durante el proceso de cultivo de café. (Verghese, Horne, \& Carre, 2010)

De este modo, desde los autores se plantea una prueba experimental con cada uno de estos desechos orgánicos con el papel reciclado para corroborar algunas de estas hipótesis y, en consecuencia, dar mayor peso como viabilidad a la propuesta del separador y del protector del vaso. Adicionalmente, se pueden plantear pruebas alternas con otros materiales orgánicos en debido caso de no funcionar dicha hipótesis. 


\section{Proceso productivo}

En la Figura 7, se puede visualizar todo el proceso por el cual será sometido cada producto, y así llegar al planteamiento deseado en este capítulo. Bajo lo anterior, se tiene previsto hacer un pequeño cambio respecto a la cadena productiva del café original, en donde el pergamino del café sea el punto de partida para empezar el proceso de fabricación del separador y del protector de vasos. Su comienzo se da con el secado, dicho proceso se caracteriza por su prolongado tiempo de espera por ello, los campesinos productores mientras tanto tienen la posibilidad de realizar una recolección por los alrededores de donde reside, ya sea que se transporte en un automóvil, cicla o caminando, lo cual genera una emisión de CO2 al ambiente si se usa un automóvil, o en caso contrario esta emisión se elimina del proceso productivo.

Tras haber hecho la respectiva recolección, el paso siguiente es recortar toda la materia recogida (cartón, hojas usadas o periódico) para transformarla en retazos más fáciles de transportar hacia los lugares dispuestos para el mojado y triturado. Para el mojado, es necesario que el papel se vuelva más blando y más fácil de triturar, en este proceso se debe deja en reposo hasta determinar cómo suficientemente "mojado" o lo suficientemente húmedo el cartón o hojas de papel. Posteriormente, se dispone del material dentro de una licuadora junto al pergamino de café y generar la transformación de este material, durante este proceso se busca generar una mezcla uniforme por medio de un tiempo e intensidad determinada para generar la materia prima deseada.

Al tener los lotes de la solución, estos deben ser introducidos en un contenedor vacío. Posteriormente, se deben utilizar matrices o molde y contra molde con la forma deseada para ser introducidos en aquel contenedor con la solución, de tal modo, que al retirarlo de este saldrá la lámina plana con el grosor determinado. Cada una de éstas debe ser puesta ante una cama, la cual permita generar presiones por ambas caras y así generar un acabado liso en las superficies. Seguidamente se debe hacer un respectivo secado de la solución, la cual es dejada al sol para absorber la humedad. También es recomendable usar algún retazo de ropa seco para ayudar a secarlo más rápido. Este proceso completo puede durar entre cinco y seis días. 
Por otra parte, una de las salidas más críticas de todo el proceso, es el agua residual que se utilizó para la humedecer los recortes y preparar el material en las bandejas. Respecto a esta situación, se pueden generar otros aprovechamientos como agua para seguir humedeciendo el papel o en la descarga de los sanitarios, en otras palabras, se pueden seguir aprovechando estas salidas de los procesos productivos. Cuando ya se haya secado la lámina, se debe separar cuidadosamente de la superficie plana para disponer ante el proceso de recorte de las formas y finalizar con el producto listo para el apilamiento y la venta.

\section{Proceso productivo de los separadores y de los protectores}

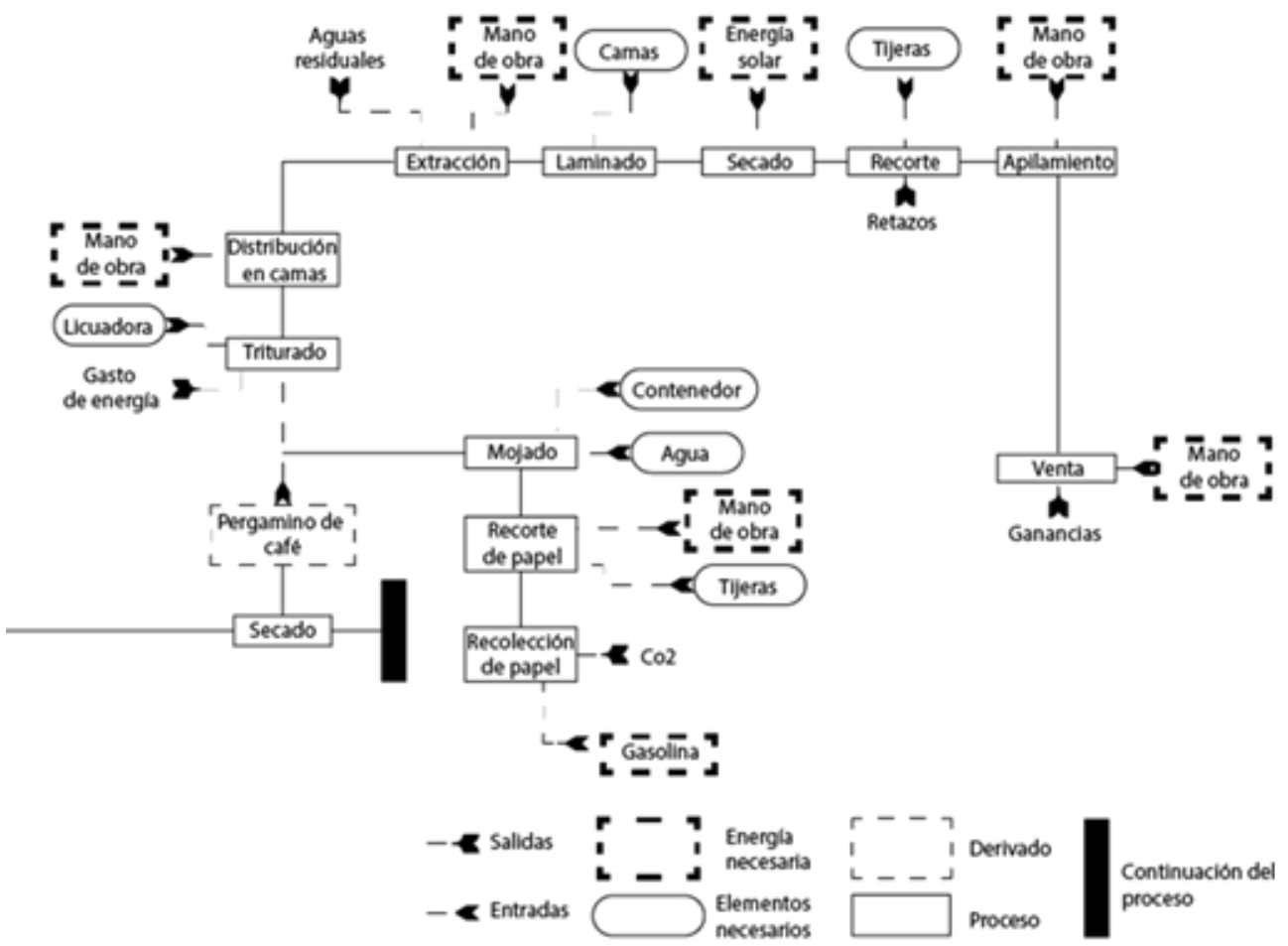

Figura 7. Proceso productivo de las propuestas.

Fuente: elaboración basada en la cadena productiva del café, figura 6.

Como complemento de la propuesta, se busca adicionalmente informar al consumidor final sobre los productos que apoya y el impacto a generar para los campesinos productores; y de este modo, se promueve una economía o consumo más consciente en donde se diferencie la variedad de productos, no solo por sus componentes o características sino por el 
impacto positivo a nivel macro al apoyar dichos productos, en consecuencia, el aporte a la cultura de la sostenibilidad desde sus tres dimensiones (Ávila, 2016).

\section{Conclusiones}

Si en la agricultura familiar se logra una producción sustentable ambientalmente como elemento básico para la diferenciación y el posicionamiento de los productos, y a partir de allí, se define una estrategia de comunicación enfocada en los consumidores responsables que estén dispuestos a pagar un precio más alto en el mercado y se eligen o se crean canales de distribución que lleguen a ellos, esto motivará a los productores a mantener su producción amigable con el medio ambiente, lo que tiene un impacto en la conservación de los recursos naturales y en la sociedad misma.

El hecho de plantear productos desde una visión del diseño sustentable, como se vio durante este capítulo, da una verdadera muestra de que se pueden realizar y dar un cambio en la economía actual, no obstante, el verdadero significado de cambio es entendido como mejorar las condiciones de vida de los más desfavorecidos, eliminando el sesgo consumista y fortaleciendo una economía solidaria que está siendo más consciente dentro de las sociedades.

La utilidad de apropiar y aplicar la perspectiva o paradigma de la sostenibilidad en proyectos proyectuales de diseño, generación de empresas o reestructuración empresarial tiene un gran potencial por el impacto que puede generar, no solo desde lo ambiental, sino también desde lo social y económico, porque se generan redes equitativas o justas en el mercado en toda la cadena productiva, abriendo la puerta a nuevos modelos de negocio que desde sus acciones muestren y concienticen al consumidor del papel de los productos, productores, empresas, servicios, etc., con la meta de promover una cultura de lo sensato (sostenible) para el mundo actual.

Con el fin de darle continuidad a este trabajo, se propone que el diseño se ponga a prueba en campo en fincas cafeteras, se analicen los costos de producción y, a partir de las problemáticas encontradas, se proponga el ajuste de los pasos del proceso de producción. 


\section{Referencias}

Aguayo, F., Peralta, M., Lama, J., \& Soltero, V. (2013). Ecodiseño. Ingeniería sostenible de la cuna a la cuna (C2C). Ciudad de México: Alfaomega.

Anacafé. Los subproductos del café. Retrieved from https://www.anacafe. org/glifos/index.php/BeneficioHumedo_Subproductos

Anacafé. Remoción del mucílago. Retrieved from http://www.anacafe.org/ glifos/index.php/BeneficiadoHumedo_Mucilago

Avila, E. (2016). Sustentabilidade e aprendizagens. Paper presented at the ELAUS, San Miguel de Tucumán, Argentina.

Bernal, M., \& Ticora, L. (2008). Manual de cartón corrugado Procesos manuales. In Materiales y técnicas para la representación de ideas de diseño. Bogotá: Pontificia Universidad Javeriana.

Bravo, D. (2015). Diseño de un empaque sustentable para la marca de trajes de baño Aquamor Swimwear, Municipio El Hatillo.

De Roux Rengifo, F. S. J. (2010). Construir Región y Paz. Revista Académica e Institucional de la Universidad Católica de Pereira. Retrieved from http://biblioteca.ucp.edu.co/ojs/index.php/paginas/article/ view/137/106

FAO. (2017). Agricultura Familiar - Antecedentes. Retrieved from http:// www.fao.org/family-farming/background/es/

García, R., \& Olaya, É. (2006). CARACTERIZACIÓN DE LAS CADENAS DE VALOR Y ABASTECIMIENTO DEL SECTOR AGROINDUSTRIAL DEL CAFÉ. In.

Geonova. (2011). ¿Qué es un producto sustentable? Retrieved from https:// geonova.wordpress.com/2011/10/31/\%C2\%BFque-es-un-producto-sustentable/

Gutiérrez, N., Henao, J., \& Oviedo, O. (2014). Metodología para la elaboración de Pellets con subproductos de Café y Cacao. In (Vol. 28, pp. 23-26): ENTORNOS.

Jedlicka, W. (2009). Packaging sustainability: tools, systems and strategies for innovative package design: John Wiley \& Sons.

Latouche, S., \& Harpagès, D. (2011). La hora del decrecimiento (1 ed.). Barcelona: Editorial Octaedro. 
McDonough, W. B., M. (2002). Design for the Triple Top Line. Retrieved from http://www.mcdonough.com/writings/design-triple-top-line/

MDZ, M. (2017). En el Pacífico flota una isla de basura del tamaño de Mendoza. Mdz. Retrieved from http://www.mdzol.com/nota/738924en-el-pacifico-flota-una-isla-de-basura-del-tamano-de-mendoza/

Medina, R. (2005). Plásticos biodegradables. In (pp. 22-25). México: ¿Cómo ves?

Piracoca-Chaves, D., \& Frias-Navarro, R. (2016). Empaques Biodegradables. Ejercicio de investigación de un estudiante de Diseño Industrial en el marco del Semillero de Investigación ICAI de la Pontificia Universidad Javeriana. In Vol. III Encuentro Latinoamericano de Universidades Sustentables. (pp. 497-505).

Puerta, G., \& Ríos, S. (2011). Composición química del mucílago de café, según el tiempo de fermentación y refrigeración. In (pp. 23-40).

Rincón, O. (2017). Ergonomía y procesos de diseño: Consideraciones metodológicas para el desarrollo de sistemas y productos. Bogotá: Pontificia Universidad Javeriana.

Verghese, K. L., Horne, R., \& Carre, A. (2010). PIQET: the design and development of an online 'streamlined' LCA tool for sustainable packaging design decision support. The International Journal of Life Cycle Assessment, 15(6), 608-620 doi:10.1007/s11367-010-0193-2 